\title{
Transversely Isotropic Poroelasticity Arising from Thin Isotropic Layers
}

\author{
J.G. Berryman
}

This paper was prepared for submittal to the Institute for Mathematics and Its Applications Workshop on Percolation and Random Media Minneapolis, $M N$

November 12-16, 1995

This is a preprint of a paper intended for publication in a journal or proceedings. Since changes may be made before publication, this preprint is made available with the understanding that it will not be cited or reproduced without the permission of the author. 


\section{DISCLAIMER}

This document was prepared as an account of work sponsored by an agency of the United States Government. Neither the United States Government nor the University of California nor any of their employees, makes any warranty, express or implied, or assumes any legal liability or responsibility for the accuracy, completeness, or usefulness of any information, apparatus, product, or process

disclosed, or represents that its use would not infringe privately owned rights. Reference herein to any specific commercial product, process, or service by trade name, trademark, manufacturer, or otherwise, does not necessarily constitute or imply its endorsement, recommendation, or favoring by the United States Government or the University of California. The views and opinions of authors expressed herein do not necessarily state or reflect those of the United States Government or the University of California, and shall not be used for advertising or product endorsement purposes. 


\begin{abstract}
Percolation phenomena play central roles in the field of poroelasticity, where two distinct sets of percolating continua intertwine. A connected solid frame forms the basis of the elastic behavior of a poroelastic medium in the presence of confining forces, while connected pores permit a percolating fluid (if present) to influence the mechanical response of the system from within. The present paper discusses isotropic and anisotropic poroelastic media and establishes general formulas for the behavior of transversely isotropic poroelasticity arising from laminations of isotropic components. The Backus averaging method is shown to provide elementary means of constructing general formulas. The results for confined fluids are then compared with the more general Gassmann formulas that must be satisfied by any anisotropic poroelastic medium and found to be in complete agreement.
\end{abstract}

\title{
1 Introduction
}

When viewed from a point close to the surface of the Earth, the structure of the Earth is often idealized as being that of a layered or laminated medium with essentially homogeneous physical properties within each layer. Such an idealization has a long history and is well represented by famous textbooks such as Ewing et al. [1957], Brekhovskikh [1980], and White [1983]. The importance of anisotropy due to fine layering (i.e., layer thicknesses small compared to the wavelength of the seismic or other waves used to probe the Earth) has been realized more recently, but efforts in this area are also well represented in the literature by the work of Postma [1955], Backus [1962], Berryman [1979], Schoenberg and Muir [1987], Anderson [1989], and many others.

In a completely different context, because of the relative ease with which their effective properties may be computed, finely layered composite laminates have been used for theoretical purposes to construct idealized but, in principle, realizable materials to test the optimality of various rigorous bounds on the effective properties of general composites. This line of research includes the work of Tartar [1976], Zhikov et al. [1979], Tartar [1985], Francfort and Murat [1986], Kohn and Milton [1986], Lurie and Cherkaev [1986], Milton [1986], Avellaneda [1987], Milton [1990], and deBotton and Castañeda [1992], among others.

There is a great deal of current interest in the anisotropy of Earth materials, and especially so when there is fluid present in pores and fractures in the Earth. Fluids of economic interest to the oil industry are typically oil, gas, and water, while fluids of interest in environmental applications are generally the same but for different reasons. Environmental concerns often center around fluid contaminants which may be in the form of oil or gas, or could be other undesirable organic materials in ground water. Brines (salt-laden waters) or steam may be used to flush other fluids out of the ground, whether for economic purposes or for environmental cleanup. Thus, it is important to understand the role of pore fluids in determining effective constants of such materials, and since we are usually dealing with Earth materials the fine layering or laminate model again plays a significant role in the analysis.

In this work, we will study some simple means of estimating the effects of fluids on elastic constants and in particular we will derive formulas for anisotropic poroelastic constants using a straightforward generalization of the method of Backus [1962] for determining the effective constants of a laminated elastic material. There has been some prior work in this area by Norris 
[1993], Gurevich and Lopatnikov [1995], and others. The main distinction between these earlier approaches and ours arises from our desire to understand the transition from elastic analysis to poroelastic analysis and make this transition as transparent as possible, whereas the earlier work in this area has started with poroelasticity as given and then applies a generalization of Backus' approach to the lamination analysis. Finally, we should point out that methods similar to the ones to be presented here could as easily (more easily!) be applied in the same context to the problem of determining percolation for fluid flow or effective fluid permeability (Darcy's constant) and that would be of some interest in these applications as well, but we will focus only on the elastic/poroelastic behavior in the present effort.

\section{Notation for Elastic Analysis}

In tensor notation, the relationship between components of stress $\sigma_{i j}$ and strain $u_{k, l}$ is given by

$$
\sigma_{i j}=c_{i j k l} u_{k, l}
$$

where $c_{i j k l}$ is the stiffness tensor, and repeated indices on the right hand side of (3) are summed. In (1), $u_{k}$ is the $k$ th Cartesian component of the displacement vector $\mathbf{u}$, and $u_{k, l}=\partial u_{k} / \partial x_{l}$. Whereas for an isotropic elastic medium the stiffness tensor has the form

$$
c_{i j k l}=\lambda \delta_{i j} \delta_{k l}+\mu\left(\delta_{i k} \delta_{j l}+\delta_{i l} \delta_{j k}\right)
$$

depending on only two parameters (the Lamé constants, $\lambda$ and $\mu$ ), this tensor can have up to 21 independent constants for general anisotropic elastic media. The stiffness tensor has pairwise symmetry in its indices such that $c_{i j k l}=c_{j i k l}$ and $c_{i j k l}=c_{i j l k}$, which will be used later to simplify the resulting equations.

The general equation of motion for elastic wave propagation through an anisotropic medium is given by

$$
\rho \ddot{u}_{i}=\sigma_{i j, j}=c_{i j k l} u_{k, l j}
$$

where $\ddot{u}_{i}$ is the second time derivative of the $i$ th Cartesian omponent of the displacement vector $\mathbf{u}$ and $\rho$ is the density (assumed constant). Equation (3) is a statement that the product of mass times acceleration of a particle is determined by the internal stress force $\sigma_{i j, j}$. For the present purposes, we are more interested in the quasistatic limit of this equation, in which case the left-hand side of ( 3 ) vanishes and the equation to be satisfied is just the force equilibrium equation

$$
\sigma_{i j, j}=0 .
$$

A commonly used simplification of the notation for elastic analysis is given by introducing the strain tensor, where

$$
e_{i j}=\frac{1}{2}\left(u_{i, j}+u_{j, i}\right)=\frac{1}{2}\left(\frac{\partial u_{i}}{\partial x_{j}}+\frac{\partial u_{j}}{\partial x_{i}}\right) .
$$


Then, using one version of the Voigt convention, in which the pairwise symmetries of the stiffness tensor indices are used to reduce the number of indices from 4 to 2 using the rules $11 \rightarrow 1$, $22 \rightarrow 2,33 \rightarrow 3,23$ or $32 \rightarrow 4,13$ or $31 \rightarrow 5$, and 12 or $21 \rightarrow 6$, we have

$$
\left(\begin{array}{l}
\sigma_{11} \\
\sigma_{22} \\
\sigma_{33} \\
\sigma_{23} \\
\sigma_{31} \\
\sigma_{12}
\end{array}\right)=\left(\begin{array}{llllll}
c_{11} & c_{12} & c_{13} & & & \\
c_{12} & c_{22} & c_{23} & & & \\
c_{13} & c_{23} & c_{33} & & & \\
& & & 2 c_{44} & & \\
& & & & 2 c_{55} & \\
& & & & & 2 c_{66}
\end{array}\right)\left(\begin{array}{l}
e_{11} \\
e_{22} \\
e_{33} \\
e_{23} \\
e_{31} \\
e_{12}
\end{array}\right)
$$

Although the Voigt convention introduces no restrictions on the stiffness tensor, we have chosen to limit discussion to the form in (6), which is not completely general. Of the 36 coefficients (of which 21 are generally independent), we choose to treat only those cases for which the 12 coefficients shown (of which nine are generally independent) are nonzero. This form includes all orthorhombic, cubic, hexagonal, and isotropic systems, while excluding triclinic, monoclinic, trigonal, and some tetragonal systems, since each of the latter contains additional off-diagonal constants that may be nonzero. Nevertheless, we will restrict our discussion to (6) or to the still simpler case of transversely isotropic (TI) materials.

For TI materials whose symmetry axis is in the $x_{3}$ direction, another common choice of notation is $c_{11}=c_{22} \equiv a, c_{12} \equiv b, c_{13}=c_{23} \equiv f, c_{33} \equiv c, c_{44}=c_{55} \equiv l$, and $c_{66} \equiv m$. There is also one further constraint on the constants that $a=b+2 m$, following from rotational symmetry in the $x_{1} x_{2}$-plane. In such materials, (6) may be replaced by

$$
\left(\begin{array}{l}
\sigma_{11} \\
\sigma_{22} \\
\sigma_{33} \\
\sigma_{23} \\
\sigma_{31} \\
\sigma_{12}
\end{array}\right)=\left(\begin{array}{llllll}
a & b & f & & & \\
b & a & f & & & \\
f & f & c & & & \\
& & & 2 l & & \\
& & & & 2 l & \\
& & & & & 2 m
\end{array}\right)\left(\begin{array}{l}
e_{11} \\
e_{22} \\
e_{33} \\
e_{23} \\
e_{31} \\
e_{12}
\end{array}\right)
$$

in which the matrix has the same symmetry as hexagonal systems and of which isotropic symmetry is a special case (having $a=c=\lambda+2 \mu, b=f=\lambda$, and $l=m=\mu$ ).

\section{Backus Averaging of Fine Elastic Layers}

Backus [1962] presents an elegant method of producing the effective constants for a finely layered medium composed of either isotropic or anisotropic elastic layers. For simplicity, we will assume that the layers are isotropic, in which case the equation relating elastic stresses $\sigma_{i j}$ to elastic strains $e_{i j}$ is given by

$$
\left(\begin{array}{l}
\sigma_{11} \\
\sigma_{22} \\
\sigma_{33} \\
\sigma_{23} \\
\sigma_{31} \\
\sigma_{12}
\end{array}\right)=\left(\begin{array}{cccccc}
\lambda+2 \mu & \lambda & \lambda & & & \\
\lambda & \lambda+2 \mu & \lambda & & & \\
\lambda & \lambda & \lambda+2 \mu & & & \\
& & & 2 \mu & & \\
& & & & 2 \mu & \\
& & & & & 2 \mu
\end{array}\right)\left(\begin{array}{l}
e_{11} \\
e_{22} \\
e_{33} \\
e_{23} \\
e_{31} \\
e_{12}
\end{array}\right) .
$$


The key idea presented by Backus is that these equations can be rearranged into a form where rapidly varying coefficients multiply slowly varying stresses or strains. For simple layering, we know physically (and can easily prove mathematically) that the normal stress and the tangential strains must be continuous at the boundaries between layers. If the layering direction is the $z$ or $x_{3}$ direction as is the normal choice in the acoustics and geophysics literature, then $\sigma_{33}, \sigma_{23}, \sigma_{31}$, $\epsilon_{11}, \epsilon_{22}$, and $e_{12}$ are continuous and in fact constant throughout such a laminated material. If the constancy of $e_{11}, e_{22}$, and $e_{12}$ were not so, the layers would necessarily experience relative slip; while if the constancy of $\sigma_{33}, \sigma_{23}$, and $\sigma_{31}$ were not so, then there would be force gradients across boundaries necessarily resulting in nonstatic material response to the lack of force equilibrium.

By making use of this elegant idea, we arrive at the following equation

$$
\left(\begin{array}{c}
\sigma_{11} \\
\sigma_{22} \\
-e_{33} \\
e_{23} \\
e_{31} \\
\sigma_{12}
\end{array}\right)=\left(\begin{array}{cccccc}
\frac{4 \mu(\lambda+\mu)}{\lambda+2 \mu} & \frac{2 \lambda \mu}{\lambda+2 \mu} & \frac{\lambda}{\lambda+2 \mu} & & & \\
\frac{2 \lambda \mu}{\lambda+2 \mu} & \frac{4 \mu(\lambda+\mu)}{\lambda+2 \mu} & \frac{\lambda}{\lambda+2 \mu} & & & \\
\frac{\lambda}{\lambda+2 \mu} & \frac{\lambda}{\lambda+2 \mu} & -\frac{1}{\lambda+2 \mu} & & & \\
& & & \frac{1}{2 \mu} & & \\
& & & & \frac{1}{2 \mu} & \\
& & & & & 2 \mu
\end{array}\right)\left(\begin{array}{l}
e_{11} \\
e_{22} \\
\sigma_{33} \\
\sigma_{23} \\
\sigma_{31} \\
e_{12}
\end{array}\right)
$$

which can be averaged essentially by inspection. Equation (9) can be viewed as a Legendre transform of the original equation, to a different set of dependent/independent variables in which both vectors have components with mixed physical significance, some being stresses and some being strains. Otherwise these equations are completely equivalent to the original ones in (8).

Performing the layer average using the symbol $\langle\cdot\rangle$, assuming as mentioned previously that the variation is along the $z$ or $x_{3}$ direction, we find, using the notation of (7),

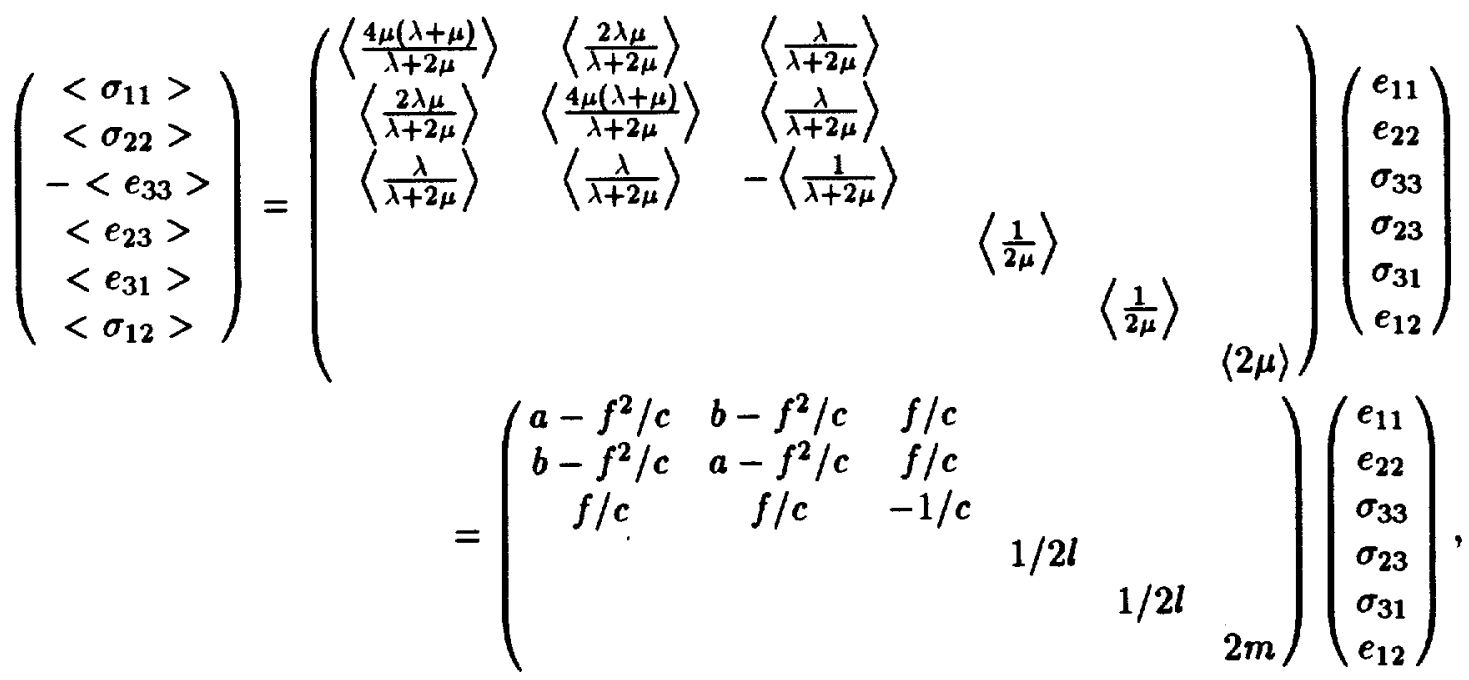

which can then be solved to yield the expressions

$$
\begin{gathered}
a=\left\langle\frac{\lambda}{\lambda+2 \mu}\right\rangle^{2}\left\langle\frac{1}{\lambda+2 \mu}\right\rangle^{-1}+4\left\langle\frac{\mu(\lambda+\mu)}{\lambda+2 \mu}\right\rangle, \\
b=\left\langle\frac{\lambda}{\lambda+2 \mu}\right\rangle^{2}\left\langle\frac{1}{\lambda+2 \mu}\right\rangle^{-1}+2\left\langle\frac{\lambda \mu}{\lambda+2 \mu}\right\rangle,
\end{gathered}
$$




$$
\begin{gathered}
c=\left\langle\frac{1}{\lambda+2 \mu}\right\rangle^{-1} \\
f=\left\langle\frac{\lambda}{\lambda+2 \mu}\right\rangle\left\langle\frac{1}{\lambda+2 \mu}\right\rangle^{-1} \\
l=\left\langle\frac{1}{\mu}\right\rangle^{-1}
\end{gathered}
$$

and

$$
m=\langle\mu\rangle
$$

Equations (11)-(16) are the well-known results of Backus [1962] for layering of isotropic elastic materials. One very important fact that is known about these equations is that they reduce to isotropic results, having $a=c, b=f$, and $l=m$, if the shear modulus $\mu$ is a constant, regardless of the behavior of $\lambda$. Another fact that can easily be checked is that $a=b+2 m$, which is a general condition that must be satisfied for all transversely isotropic materials and shows that there are only five independent constants.

\section{Porous Elastic Materials Containing Fluids}

Now we want to broaden our outlook and suppose that the materials composing the laminate are not homogeneous isotropic elastic materials, but rather elastic materials containing voids or pores. The pores may be either air-filled, or alternatively they may be partially or fully saturated with a liquid, a gas, or a fluid mixture. For simplicity, we will suppose here that the pores are either air-filled or they may be fully saturated with some other homogeneous fluid. When the porous layers are air-filled, it is generally adequate to assume that the analysis of the preceding section holds, but with the new interpretation that the Lamé parameters are those for the porous elastic medium in the absence of saturating fluids. The resulting effective constants $\lambda_{d r}$ and $\mu_{d r}$ are then said to be those for "dry" - or somewhat more accurately "drained" - conditions. These constants are also sometimes called the "frame" constants, to distinguish them from the constants associated with the solid materials composing the frame which are often called the "grain" or "mineral" constants.

One simplification that arises immediately is due to the fact that the presence of pore fluids has no mechanical effect on the shear moduli, so $\mu_{d r}=\mu$. There may be other effects on the shear moduli due to the presence of pore fluids, such as softening of cementing materials or expansion of interstitial clays, which we will term "chemical" effects to distinguish them from the purely mechanical effects to be considered here. We neglect all such chemical effects in the following analysis. This means that the lamination analysis for the effective shear moduli (since it is uncoupled from the analysis involving $\lambda$ ) does not change in the presence of fluids. Thus, equations (15) and (16) continue to apply for the poroelastic problem, and we can therefore simplify our system of equations in order to focus on the parts of the analysis that do change in the presence of fluids. 
The presence of a saturating pore fluid introduces the possibility of an additional control field and an additional type of strain variable. The pressure $p_{f}$ in the fluid is the new field parameter that can be controlled. Allowing sufficient time for global pressure equilibration will permit us to consider $p_{f}$ to be a constant throughout the percolating (connected) pore fluid, while restricting the analysis to quasistatic processes. The change $\zeta$ in the amount of fluid mass contained in the pores is the new type of strain variable, measuring how much of the original fluid in the pores is squeezed out during the compression of the pore volume while including the effects of compression or expansion of the pore fluid itself due to changes in $p_{f}$. It is most convenient to write the resulting equations in terms of compliances rather than stiffnesses, so the basic equation to be considered takes the form:

$$
\left(\begin{array}{c}
\epsilon_{11} \\
e_{22} \\
e_{33} \\
-\zeta
\end{array}\right)=\left(\begin{array}{cccc}
s_{11} & s_{12} & s_{12} & -\beta \\
s_{12} & s_{11} & s_{12} & -\beta \\
s_{12} & s_{12} & s_{11} & -\beta \\
-\beta & -\beta & -\beta & \gamma
\end{array}\right)\left(\begin{array}{c}
\sigma_{11} \\
\sigma_{22} \\
\sigma_{33} \\
-p_{f}
\end{array}\right) .
$$

The constants appearing in the matrix on the right hand side will be defined in the following two paragraphs. It is important to write the equations this way rather than using the inverse relation in terms of the stiffnesses, because the compliances $s_{i j}$ appearing in (17) are simply related to the drained constants $\lambda_{d r}$ and $\mu_{d r}$ in the same way they are related in normal elasticity, whereas the individual stiffnesses obtained by inverting the equation in (17) must contain coupling terms through the parameters $\beta$ and $\gamma$ that depend on the pore and fluid compliances. Thus, we find easily that

$$
s_{11}=\frac{1}{E_{d r}}=\frac{\lambda_{d r}+\mu}{\mu\left(3 \lambda_{d r}+2 \mu\right)}
$$

and

$$
s_{12}=-\frac{\nu_{d r}}{E_{d r}}
$$

where the drained Young's modulus $E_{d r}$ is defined by the second equality of (18) and the drained Poisson's ratio is determined by

$$
\nu_{d r}=\frac{\lambda_{d r}}{2\left(\lambda_{d r}+\mu\right)}
$$

When the external stress is hydrostatic so $\sigma=\sigma_{11}=\sigma_{22}=\sigma_{33}$, the equation (17) telescopes down to

$$
\left(\begin{array}{c}
e \\
-\zeta
\end{array}\right)=\left(\begin{array}{cc}
1 / K_{d r} & -\alpha / K_{d r} \\
-\alpha / K_{d r} & \alpha / B K_{d r}
\end{array}\right)\left(\begin{array}{c}
\sigma \\
-p_{f}
\end{array}\right)
$$

where $e=e_{11}+e_{22}+e_{33}, K_{d r}=\lambda_{d r}+\frac{2}{3} \mu$ is the drained bulk modulus, $\alpha=1-K_{d r} / K_{m}$ is the Biot-Willis parameter [Biot and Willis, 1957] with $K_{m}$ being the bulk modulus of the solid minerals present, and Skempton's pore-pressure buildup parameter $B$ [Skempton, 1954] is given by

$$
B=\frac{1}{1+K_{p}\left(1 / K_{f}-1 / K_{m}\right)}
$$


New parameters appearing in (22) are the bulk modulus of the pore fluid $K_{f}$ and the pore modulus $K_{p}=\alpha / \phi K_{d r}$ where $\phi$ is the porosity. The expressions for $\alpha$ and $B$ can be generalized slightly by supposing that the solid frame is composed of more than one constituent, in which case the $K_{m}$ appearing in the definition of $\alpha$ is replaced by $K_{s}$ and the $K_{m}$ appearing explicitly in (22) is replaced by $K_{\phi}$ [see Brown and Korringa, 1975; Rice and Cleary, 1976; Berryman and Milton, 1991; Berryman and Wang, 1995]. This is an important additional complication [Berge and Berryman, 1995], but one that we choose not to pursue here.

Comparing (17) and (21), we find easily that

$$
\beta=\frac{\alpha}{3 K_{d r}}
$$

and

$$
\gamma=\frac{\alpha}{B K_{d r}}
$$

With all the constants defined now in terms of measureable quantities, we can continue with the analysis that generalizes the Backus [1962] approach to computing the layer averages. It should be clear at this point that the appropriate Legendre transformed equations are

$$
\begin{aligned}
&\left(\begin{array}{c}
\sigma_{11} \\
\sigma_{22} \\
-e_{33} \\
\zeta
\end{array}\right)= \\
&\left(\begin{array}{cccc}
E /\left(1-\nu^{2}\right) & \nu E /\left(1-\nu^{2}\right) & \nu /(1-\nu) & \beta E /(1-\nu) \\
\nu E /\left(1-\nu^{2}\right) & E /\left(1-\nu^{2}\right) & \nu /(1-\nu) & \beta E /(1-\nu) \\
\nu /(1-\nu) & \nu /(1-\nu) & -\left(1-\nu-2 \nu^{2}\right) /(1-\nu) E & \beta(1+2 \nu) /(1-\nu) \\
\beta E /(1-\nu) & \beta E /(1-\nu) & \beta(1+2 \nu) /(1-\nu) & -\left[\gamma-2 \beta^{2} E /(1-\nu)\right]
\end{array}\right), \\
&
\end{aligned}
$$

with the fast variables on the left and the slow variables (actually constant) in the vector on the right. Signs have been chosen so the matrix is symmetric. We have also dropped the subscript $d r$ from the drained constants $\nu$ and $E$ in (25) as there should be no confusion. Note that the $3 \times 3$ submatrix in the upper left is identical to that in (9) after the change in notation from $\lambda, \mu$ to $E, \nu$ is taken into account.

Once we have this equation, the averaging is trivial. If the assumed form of the resulting equations is taken - in analogy to (7) and consistent with the general structure of the matrix in (25) - to be

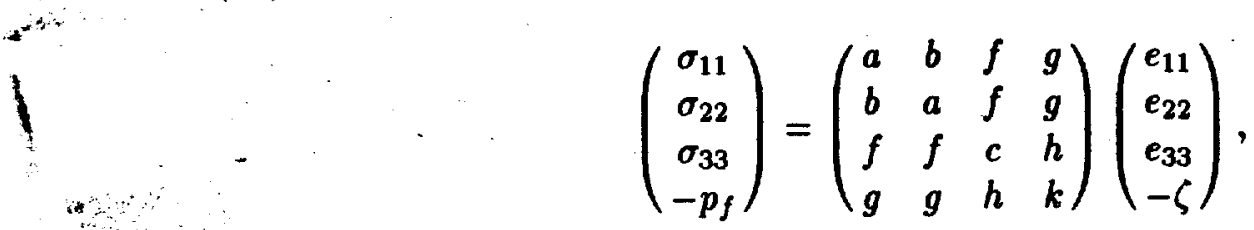


then the resulting rearrangement of these equations is

$$
\left(\begin{array}{c}
<\sigma_{11}> \\
<\sigma_{22}> \\
-\left\langle e_{33}>\right. \\
<\zeta>
\end{array}\right)=\left(\begin{array}{cccc}
a-x & b-x & y & z \\
b-x & a-x & y & z \\
y & y & u & v \\
z & z & v & w
\end{array}\right)\left(\begin{array}{c}
e_{11} \\
e_{22} \\
\sigma_{33} \\
-p_{f}
\end{array}\right),
$$

where

$$
x=\frac{f^{2} k-2 f g h+c g^{2}}{c k-h^{2}}, \quad y=\frac{f k-g h}{c k-h^{2}}, \quad z=\frac{c g-f h}{c k-h^{2}}
$$

and

$$
u=-\frac{k}{c k-h^{2}}, \quad v=\frac{h}{c k-h^{2}}, \quad w=-\frac{c}{c k-h^{2}} .
$$

It is not difficult to check that these equations reduce correctly to the earlier ones if we first set $g=h=0$ and then let $k \rightarrow 0$.

Now all the matrix elements appearing in (27) are obtained directly by averaging (25) and therefore are assumed known. We will not list all of these relations as they should be clear from the expression already given, but to provide two examples we note that

$$
w=2\left\langle\frac{\beta^{2} E}{1-\nu}\right\rangle-\langle\gamma\rangle \quad \text { and } \quad z=\left\langle\frac{\beta E}{1-\nu}\right\rangle .
$$

Given all these equations, it is then straightforward to invert for the desired final expressions:

$$
\begin{gathered}
a=\left\langle\frac{E}{1-\nu^{2}}\right\rangle+x, \\
b=\left\langle\frac{\nu E}{1-\nu^{2}}\right\rangle+x, \\
c=-\frac{w}{u w-v^{2}}, \\
f=c y+h z, \\
g=h y+k z, \\
h=\frac{v}{u w-v^{2}},
\end{gathered}
$$

and

$$
k=-\frac{u}{u w-v^{2}}
$$


The order in which the computations are done in practice is this: first compute $c, h$, and $k$; next compute $f$ and $g$; then compute $x$ using (28); finally compute $a$ and $b$.

The results show that, whereas transverse isotropy in elastic materials due to layering produces five independent constants (recall that $a=b+2 m$ in general for transverse isotropy), transverse isotropy in poroelastic materials results in eight independent constants $(a=b+2 m$ still holds for poroelasticity as is easily shown from our formulas). When performing the averaging based on (25), we see that all the new terms in the matrix depend on averages of the poroelastic constant $\beta$ which is proportional to the Biot-Willis parameter and therefore related to effective stress [the relative importance of external and internal loading - see (21)]. However, only the new diagonal term $w$ depends directly on the bulk modulus $K_{f}$ of the pore fluid through $\gamma$. It follows that, when we solve for the effective constants, we will find that $w$ influences all these effective constants. So the presence of pore fluid can significantly affect the pressure dependence of such materials, while having little or no effect on the shear response.

This completes the analysis of the constants for transverse isotropy in poroelasticity arising from thin layering of isotropic elastic and porous materials. Now we should check that these results are consistent with known general results for anisotropic poroelasticity [Gassmann, 1951; Brown and Korringa, 1975].

\section{Relations for Anisotropy in Poroelastic Materials}

Gassmann [1951] and Brown and Korringa [1975] have considered the problem of obtaining effective constants for anisotropic poroelastic materials when the pore fluid is confined within the pores. The confinement condition amounts to the constraint that the increment of fluid content $\zeta=0$, while the external loading $\sigma$ is changed and the pore-fluid pressure $p_{f}$ is allowed to respond as necessary and equilibrate.

To provide a simple derivation of the Gassmann equation for anisotropic materials, we consider

$$
\left(\begin{array}{c}
e_{11} \\
e_{22} \\
e_{33} \\
-\zeta
\end{array}\right)=\left(\begin{array}{cccc}
s_{11} & s_{12} & s_{13} & -\beta_{1} \\
s_{12} & s_{22} & s_{23} & -\beta_{2} \\
s_{13} & s_{23} & s_{33} & -\beta_{3} \\
-\beta_{1} & -\beta_{2} & -\beta_{3} & \gamma
\end{array}\right)\left(\begin{array}{c}
\sigma_{11} \\
\sigma_{22} \\
\sigma_{33} \\
-p_{f}
\end{array}\right) .
$$

The shear terms are excluded as before since they do not interact mechanically with the fluid effects. This form is not completely general in that it includes orthorhombic, cubic, hexagonal, and all isotropic systems, but excludes triclinic, monoclinic, trigonal, and some tetragonal systems that would have some nonzero off-diagonal terms in the full elastic matrix. Also, we have assumed that the material axes are aligned with the spatial axes. But this latter assumption is not significant for the derivation that follows. Such an assumption is important when properties of laminated materials having arbitrary orientation relative to the spatial axes need to be considered, but we will not treat this more general problem here.

Before proceeding, we should discuss the significance of the matrix elements appearing in (38) briefly. In the so-called "jacketed test," a porous sample is enclosed in a thin jacketing material with a tube into the pore space to permit the fluid to flow freely in or out while maintaining constant fluid pressure. Then it is sufficient to consider the case with $p_{f}=0$. We see that it is possible under these circumstances, at least in principle, to make 12 independent 
measurements by varying $\sigma_{i i}$ 's and measuring $e_{j j}$ 's and $\zeta$. In fact measurements of drained elastic compliances are commonly made in such a manner, but it is less common for the $\beta_{i}$ 's to be measured this way. To complete the measurements, a second common test - the so-called "unjacketed test" - is performed in which a uniform pressure field is applied to the sample so that $\sigma_{11}=\sigma_{22}=\sigma_{33}=-p_{f}$. Then, by making measurements of the $e_{j j}$ 's again as $p_{f}$ varies, we measure a set of solid material compliances $\kappa_{i}$, given essentially by row sums of the matrix in (38)

$$
\kappa_{i}=\sum_{j=1}^{3} s_{i j}-\beta_{i}, \quad \text { for } \quad i=1,2,3 .
$$

These constants are expected to be related to the compliances of the mineral grains composing the porous frame; if the frame is microhomogeneous (i.e., containing a single solid constitutent), the compliances $\kappa_{i}$ will be the compliances of the mineral composing the frame such that $\sum_{i} \kappa_{i}=1 / K_{m}$, where $K_{m}$ is the bulk modulus of the mineral. If the measurement apparatus is inadequate so that the $\beta_{i}$ 's could not be determined directly in the jacketed test, then we see from (31) that they can be determined by combining results from the jacketed and unjacketed measurements on the solid compliances. The remaining constant $\gamma$ can again be measured (at least in principle) directly in the unjacketed test by making measurements on the changes in fluid content $\zeta$. An alternative to these difficult measurements is the confined test which we describe next.

If the fluid is confined, then $\zeta \equiv 0$ in (38) and $p_{f}$ becomes a linear function of $\sigma_{11}, \sigma_{22}, \sigma_{33}$. Eliminating $p_{f}$ from the resulting equations, we obtain the general expression for the strain dependence on external stress under confined conditions:

$$
\begin{array}{r}
\left(\begin{array}{l}
e_{11} \\
e_{22} \\
e_{33}
\end{array}\right)=\left[\left(\begin{array}{lll}
s_{11} & s_{12} & s_{13} \\
s_{12} & s_{22} & s_{23} \\
s_{13} & s_{23} & s_{33}
\end{array}\right)-\gamma^{-1}\left(\begin{array}{c}
\beta_{1} \\
\beta_{2} \\
\beta_{3}
\end{array}\right)\left(\begin{array}{lll}
\beta_{1} & \beta_{2} & \beta_{3}
\end{array}\right)\right]\left(\begin{array}{l}
\sigma_{11} \\
\sigma_{22} \\
\sigma_{33}
\end{array}\right) \\
\equiv\left(\begin{array}{lll}
s_{11}^{*} & s_{12}^{*} & s_{13}^{*} \\
s_{12}^{*} & s_{22}^{*} & s_{23}^{*} \\
s_{13}^{*} & s_{23}^{*} & s_{33}^{*}
\end{array}\right)\left(\begin{array}{l}
\sigma_{11} \\
\sigma_{22} \\
\sigma_{33}
\end{array}\right) .
\end{array}
$$

The $s_{i j}$ 's are fluid-drained constants, while the $s_{i j}^{*}$ 's are the fluid-confined constants.

The fundamental result obtained by both Gassmann [1951] and Brown and Korringa [1975] may be written as

$$
s_{i j}^{*}=s_{i j}-\frac{\beta_{i} \beta_{j}}{\gamma}, \quad \text { for } \quad i, j=1,2,3
$$

This expression is just the anisotropic generalization of the well-known Gassmann equation. Equation (41) has often been written in a slightly different way, by making use of the formulas (39) to eliminate the $\beta$ 's in favor of the solid and drained compliances. The principal advantage of the alternative formula is that all constants appearing explicitly can be obtained by measurements of porous frame strain, without resorting to the more difficult measurements of changes in pore fluid content.

Now it is not difficult to see that the lamination formulas derived earlier in the paper satisfy these general conditions. This simple test provides one means of checking that we did the lamination analysis correctly and also provides a convenient means of summarizing the results. 


\section{Conclusion}

In this paper, we have shown how to make the transition from analysis of laminations of elastic materials to laminations of poroelastic materials in the presence of saturating pore fluids. Backus [1962] averaging based on the simple observation that certain variables are quasistatically constant across a layered medium provides a very intuitive and mathematically transparent approach to obtaining formulas of current interest. Such results are especially important for applications to oil exploration using AVO (amplitude versus offset) since the presence or absence of the fluid component, as well as the nature of the fluid, is the critical issue and the ways in which the fluid can influence seismic reflection data need to be understood in more detail than has been possible in the past [Thomsen, 1993; Mukerji and Mavko, 1994].

\section{Acknowledgments}

I thank Graeme Milton for helpful comments and pointers to the literature on lamination analysis. Work was performed under the auspices of the U. S. Department of Energy by the Lawrence Livermore National Laboratory under contract No. W-7405-ENG-48 and supported specifically by the Geosciences Research Program of the DOE Office of Energy Research within the Office of Basic Energy Sciences, Division of Engineering and Geosciences, as part of the Advanced Computational Technology Initiative.

\section{REFERENCES}

D. L. Anderson, Theory of the Earth, Blackwell, Boston, 1989, pp. 317-319.

M. Avellaneda, Optimal bounds and microgeometries for elastic composites, SIAM J. Appl. Math. 47, 1216-1228 (1987).

G. E. Backus, Long-wave elastic anisotropy produced by horizontal layering, J. Geophys. Res., 67, 4427-4440 (1962).

P. A. Berge and J. G. Berryman, Realizability of negative pore compressibility in poroelastic ocmposites, ASME J. Appl. Mech. 62, 1053-1062 (1995).

J. G. Berryman, Long-wave elastic anisotropy in transversely isotropic media, Geophysics 44, 896-917 (1979).

J. G. Berryman and G. W. Milton, Exact results for generalized Gassmann's equations in composite porous media with two constituents, Geophysics 56, 1950-1960 (1991).

J. G. Berryman and H. F. Wang, The elastic coefficients of double-porosity models for fluid transport in jointed rock, J. Geophys. Res. 100, 24611-24627 (1995).

M. A. Biot, Mechancis of deformation and acoustic propagation in porous media, J. Appl. Phys. 33, 1482-1498 (1962).

M. A. Biot and D. G. Willis, The elastic coefficients of the theory of consolidation, J. Appl. Mech. 24, 594-601 (1957). 
L. M. Brekhovskikh, Waves in Layered Media, Academic Press, New York, 1980, pp. 81-101.

R. J. S. Brown and J. Korringa, On the dependence of the elastic properties of a porous rock on the compressibility of a pore fluid, Geophysics 40, 608-616 (1975).

G. deBotton and P. P. Castañeda, On the ductility of laminated materials, Int. J. Solids Struct. 29, 2329-2353 (1992).

W. M. Ewing, W. S. Jardetsky, and F. Press, Elastic Waves in Layered Media, McGraw-Hill, New York, 1957, pp. 238-245.

G. A. Francfort and F. Murat, Homogenization and optimal bounds in linear elasticity, Arch. Rational Mech. Anal. 94, 307-(1986).

F. Gassmann, Über die elastizität poröser medien, Veirteljahrsschrift der Naturforschenden Gesellschaft in Zürich 96, 1-23 (1951).

B. Gurevich and S. L. Lopatnikov, Velocity and attenuation of elastic waves in finely layered porous rocks, Geophys. J. Int. 121, 933-947 (1995).

R. V. Kohn and G. W. Milton, On bounding the effective conductivity of anisotropic composites, in Homogenization and Effective Moduli of Materials and Media, edited by J. L. Eriksen, D. Kinderlehrer, R. Kohn, and J.-L. Lions, Springer-Verlag, New York, 1986, pp. 97-125.

K. A. Lurie and A. V. Cherkaev, The effective characteristics of composite materials and optimal design of constructs, Advances in Mechanics (Poland) 8, 3-81 (1986). [in Russian]

G. W. Milton, Modelling the properties of composites by laminates, in Homogenization and Effective Moduli of Materials and Media, edited by J. L. Eriksen, D. Kinderlehrer, R. Kohn, and J.-L. Lions, Springer-Verlag, New York, 1986, pp. 150-174.

G. W. Milton, On characterizing the set of possible effective tensors of composites: The variational method and the translation method, Commun. Pure Appl. Math. 43, 63-125 (1990).

T. Mukerji and G. Mavko, Pore fluid effects on seismic velocity in anisotropic rocks, Geophysics 59, 233-244 (1994).

A. N. Norris, Low-frequency dispersion and attenuation in partially saturated rocks, J. Acoust. Soc. Am. 94, 359-370 (1993).

G. W. Postma, Wave propagation in a stratified medium, Geophysics 20, 780-806 (1955).

J. R. Rice and M. P. Cleary, Some basic stress diffusion solutions for fluid-saturated elastic porous media with compressible constituents, Rev. Geophys. 14, 227-241 (1976).

M. Schoenberg and F. Muir, A calculus for finely layered anisotropic media, Geophysics 54, 581-589 (1989).

1. W. Skempton, The pore-pressure coefficients $A$ and $B$, Geotechnique 4, 143-147 (1954). 
L. Tartar, Estimation de coefficients homogeneises, in Computer Methods in Applied Sciences and Engineering, R. Glowinski and J.-L. Lions (eds.), Springer-Verlag Lecture Notes in Mathematics, Springer-Verlag, Berlin, 704, 136-212 (1976).

L. Tartar, Estimation fines de coefficients homogeneises, in Ennio De Giorgi's Colloquium, P. Kree (ed.), Research Notes in Mathematics, Pitman Press, London, 125, 168-187 (1985).

L. Thomsen, Weak anisotropic reflections, in Offset-Dependent Reflectivity - Theory and Practice of AVO Analysis, J. P Castagna and M. M. Backus (eds.), Society of Exploration Geophysicists, Tulsa, Oklahoma, 1993, pp. 103-111.

J. E. White, Underground Sound: Application of Seismic Waves, Elsevier, Amsterdam, 1983.

V. V. Zhikov, S. M. Koslov, O. A. Oleinik, and K. T. Ngoan, Averaging and G-convergence of differential operators, Russian Math. Surveys 34, 69-147 (1979). 


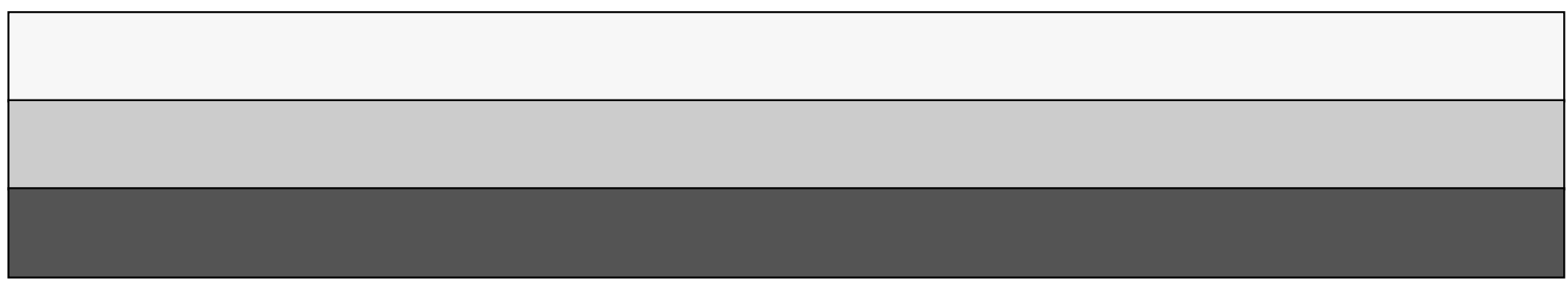

